\title{
Estratigrafía del cráter y morfología del volcán Cerro Machín, Colombia
}

\author{
Daniel Alberto Piedrahita ${ }^{1,2 *}$; Camila Aguilar-Casallass ${ }^{3}$ Eliana Arango-Palacio ${ }^{4}$; Hugo Murcia ${ }^{2,5}$; \\ Johana Gómez-Arango ${ }^{6}$
}

DOI: http://dx.doi.org/10.18273/revbol.v40n3-2018002@ @

Forma de citar: Piedrahita, D.A., Aguilar-Casallas, C., Arango-Palacio, E., Murcia, H., y Gómez-Arango, J. (2018). Estratigrafía del cráter y morfología del volcán Cerro Machín, Colombia. Boletín de Geología, 40(3), 29-48. DOI: 10.18273/revbol.v40n3-2018002.

\section{RESUMEN}

El Volcán Cerro Machín (VCM) se localiza en el flanco oriental de la Cordillera Central de Colombia a 17 km al occidente de la ciudad de Ibagué (Tolima). El VCM es un volcán Holocénico de composición dacítica, con evidencia de grandes erupciones (VEI 5). Estratigráficamente, en las paredes internas del cráter es posible observar una secuencia de depósitos de Corrientes de Densidad Piroclástica (CDPs) diluida con características de depósitos de oleadas basales. La presencia de líticos accidentales evidencia que durante la fragmentación fue involucrado el basamento. De esta manera es posible sugerir que la interacción agua/magma jugó un papel importante en la dinámica del último evento eruptivo, el cual constituye los depósitos que conforman el cráter. Análisis morfológicos evidencian un cráter de 2,4 km de diámetro con una abertura hacia el sector suroccidental, el cual alberga un domo de $490 \mathrm{~m}$ de altura que posiblemente durante su emplazamiento fue el causante de la destrucción del cráter. Aunque el VCM es sin duda un volcán poligenético (seis erupciones en los últimos 5000 años), las características sedimentológicas de los depósitos del cráter así como su morfología, sugieren una estructura monogenética de cono de toba. En conclusión, el VCM debe ser definido como un "volcán compuesto con morfología de cono de toba”.

Palabras clave: Corrientes de Densidad Piroclástica (CDPs); oleadas piroclásticas; geomorfología volcánica; volcán poligenético; cono de toba.

\section{Stratigraphy of the crater and morphology of Cerro Machín volcano, Colombia}

\begin{abstract}
The Cerro Machín Volcano (CMV) is located on the eastern flank of the Central Cordillera of Colombia, $17 \mathrm{~km}$ west of the city of Ibagué (Tolima). The CMV, a dacitic Holocene volcano, has evidence of multiple considerable eruptions (VEI 5). The inner walls of the crater display a sequence of dilute pyroclastic density currents (PDC) deposits, which contain characteristics of basal surge deposits. The presence of accidental lithics indicates that the basement of the volcano was involved in the magmatic fragmentation process. It is, thus possible to suggest that water-magma interaction played an important role in the dynamics of the last eruptive event, which formed the deposits that molded the crater. On the other hand, a morphological analysis revealed that the crater is 2.4 $\mathrm{km}$ in diameter, has an opening which faces the southwestern sector, and carries a dome of $490 \mathrm{~m}$ in height. Thus, it is interpreted that the growing dome destroyed the crater during its emplacement. Although the CMV is undoubtedly a polygenetic volcano (six eruptions in the last 5000 years), the sedimentological and morphological characteristics of the crater deposits suggest a monogenetic tuff cone structure. In conclusion, the CMV should be defined as a "composite volcano with tuff cone morphology".
\end{abstract}

Keywords: Pyroclastic density currents; pyroclastic surges; volcanic geomorphology; polygenetic volcano; tuff cone.

\footnotetext{
${ }^{1}$ Maestría en Ciencias de la Tierra, Universidad de Caldas, Manizales, Colombia. (*) danielpt_93@hotmail.com

${ }^{2}$ Instituto de Investigaciones en Estratigrafía, Universidad de Caldas, Manizales, Colombia. hugo.murcia@ucaldas.edu.co

${ }^{3}$ Programa de Geología, Universidad de Caldas, Manizales, Colombia. camiaguilar05@gmail.com

${ }^{4}$ Doctorado en Ciencias Geológicas, Universidad de Salta, Salta, Argentina. eliana.arango@ucaldas.edu.co

${ }^{5}$ Departamento de Ciencias Geológicas, Universidad de Caldas, Manizales, Colombia.

${ }^{6}$ Posgrado en Ciencias de la Tierra, Universidad Nacional Autónoma de México, Morelia, México. johanagomezarango@gmail.com
} 


\section{INTRODUCCIÓN}

En un volcán, la estructura de su cráter alberga información que evidencia los procesos de construcción a través del tiempo (e.g. tipo de depósitos); estos procesos a su vez, son la respuesta a aquellos que ocurren cuando el magma alcanza superficie (e.g. tipo de fragmentación) (Cano-Cruz y Carrasco-Núñez, 2008). Asimismo, la morfología de un cráter está relacionada con la dinámica eruptiva y la periodicidad de eventos en el volcán (Branney y Kokelaar, 2002; Kereszturi y Németh, 2012). Para volcanes poligenéticos, el cráter y en si el edificio volcánico, es el resultado de la evolución de miles e inclusive millones de años $\left(10^{3}-10^{6}\right.$ años) de construcción, mientras que para volcanes monogenéticos la estructura es evidencia de menor tiempo ( $10^{2}$ años) (Fisher y Schmincke, 1984; De Silva y Lindsay, 2015). Para ambos tipos de volcanes, los procesos de denudación generan cambios importantes en el cráter, la estructura y en sí la forma del volcán (Wood, 1980; Dóniz-Páez,
2005; Karátson et al., 2010; Kereszturi y Németh, 2012). El Volcán Cerro Machín (VCM; N 4²9', W 75²2', 2750 msnm) es uno de los volcanes que más interés despierta en el país debido a su alto potencial explosivo y gran extensión alcanzada por sus depósitos (e.g. Cortés, 2001a). Este volcán se ubica en el flanco oriental de la Cordillera Central de Colombia (FIGURA 1), y hace parte de la Provincia Volcano Tectónica San Diego Cerro Machín (PVTSC; cf. Martínez et al., 2014). El VCM se encuentra a 7,5 km del municipio de Cajamarca (Tolima), $17 \mathrm{~km}$ al occidente de la ciudad de Ibagué (Tolima), y $150 \mathrm{~km}$ al suroccidente de la capital Bogotá (Cundinamarca). El volcán se caracteriza por haber generado columnas plinianas (hasta $32 \mathrm{~km}$ de altura) generando caídas piroclásticas que alcanzaron distancias hasta de decenas de kilómetros, así como Corrientes de Densidad Piroclástica (CDPs) y lahares que fluyeron grandes distancias (e.g. Cortés, 2001a y 2001b; Méndez, 2001; Rueda, 2005; Murcia et al., 2008; 2010).

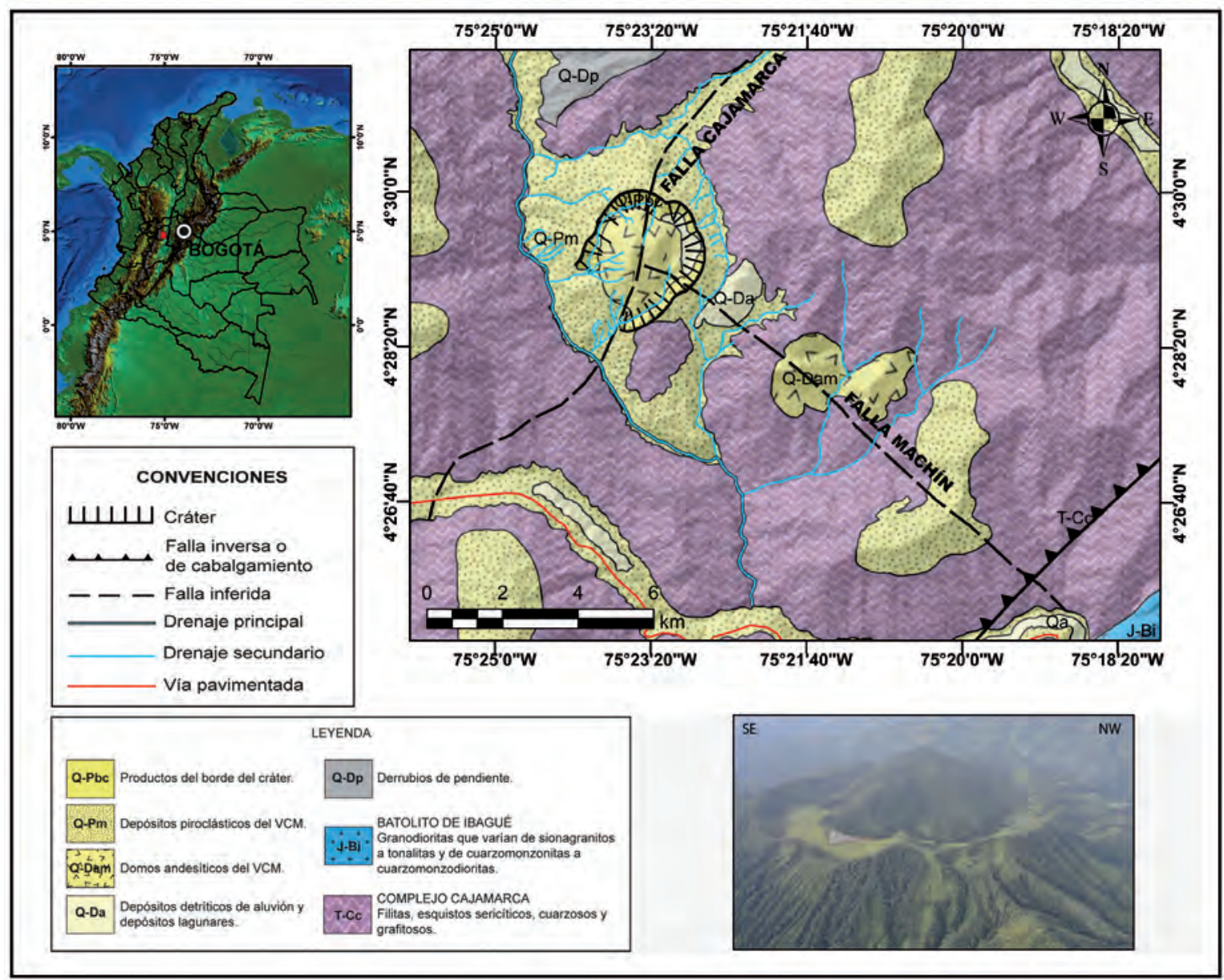

FIGURA 1. Localización del Volcán Cerro Machín (CVM) en la Cordillera Central de Colombia (recuadro rojo), la falla Cajamarca es tomada de Mosquera (1978) y la falla Machín de Cepeda et al. (1995), Mapa geológico del VCM y sus alrededores (adaptado de CHEC, 1983; Cepeda et al., 1995). Fotografía del VCM (www.sgc.gov.co). Nótese el domo intracratérico la actual "laguna" intracratérica. 
Específicamente su historia Holocénica está enmarcada en seis eventos eruptivos ocurridos durante los últimos 5000 años: Las erupciones de 5000, 4600 y 3600 años AP, se caracterizaron por generar depósitos de flujos piroclásticos de pómez y depósitos de oleadas piroclásticas producidos por erupciones plinianas; la erupción de 2600 años AP, se caracterizó por presentar depósitos de flujos piroclásticos de pómez y flujos piroclásticos de bloques y ceniza producidos por una erupción vulcaniana; la erupción de 1200 años AP, se caracterizó por generar depósitos de caída piroclástica y flujos piroclásticos de pómez producidos por una erupción pliniana; la erupción de 900 años AP, se caracterizó por generar depósitos de caída piroclástica y flujos piroclásticos de bloques y ceniza producidos por erupción vulcaniana (Rueda, 2005). A pesar del conocimiento de su dinámica eruptiva, estudios detallados acerca de la evolución del cráter actual no han sido reportados en la literatura.

El presente trabajo se enfoca en: 1) la caracterización sedimentológica y composicional de los depósitos formadores de las paredes internas del cráter actual con el objetivo de realizar una interpretación del mecanismo de fragmentación y dinámica eruptiva de la erupción que lo formó; y 2) una caracterización morfológica del edificio volcánico por medio de fotografías aéreas y de modelos de elevación digital (DEM, por sus siglas en inglés), con el objetivo de entender su evolución a través del tiempo. Este estudio pretende generar información para mejorar la comprensión actual de la evolución del volcán Cerro Machín.

\section{MARCO GEOLÓGICO}

El VCM es una de las estructuras más australes de la PVTSC, una cadena volcánica de aproximadamente $140 \mathrm{~km}$ que responde a la interacción de las placas Caribe, Nazca y Suramericana (e.g. Van der Hilst y Mann, 1994; Arcila et al., 2002; Trenkamp et al., 2002). El VCM, así como todo el vulcanismo calcoalcalino de la Cordillera Central de Colombia, hace parte de la Zona Volcánica Norte (ZVN) de los Andes (Bourdon et al., 2003). Actualmente, la PVTSC se considera como el vulcanismo más septentrional de la ZVN (Bourdon et al., 2003), con una profundidad de la placa Nazca bajo la provincia de $150 \mathrm{~km}$ aproximadamente (Monsalve y Mora, 2005). Entre las principales características estructurales de la zona se encuentra la falla de Ibagué, una estructura de tipo transcurrente dextral orientada en dirección ENE que constituye la expresión más evidente de una zona de cizalla transversal que afecta la Cordillera Central de Colombia (Diederix et al., 2006).

El VCM se encuentra construido sobre un conjunto de rocas metamórficas Permo-Triásicas y Jurásicas denominadas Complejo Cajamarca que constituyen el núcleo de la Cordillera Central de Colombia y que está conformado por una variedad de rocas producto del metamorfismo regional de medio a alto grado, principalmente de esquistos de clorita-albitaepidota, clorita-albita-actinolita, cuarzo-sericita-grafito, cuarcitas y cuarcitas biotíticas (Núñez et al., 1979; Villagómez et al., 2011; Blanco-Quintero et al., 2014). El volcán, presenta fenómenos asociados a actividad termal expresados en campos fumarólicos sobre el domo y fuentes termales tanto dentro como fuera del edificio volcánico (www.sgc.gov.vo). El Cerro Machín, es un volcán que ha sido interpretado como ubicado en la intersección de la falla Cajamarca con rumbo $\mathrm{N} 20^{\circ} \mathrm{E}$ (Mosquera, 1978) y la falla Machín con rumbo N24 W con una componente dextral y movimiento normal (Cepeda et al., 1995), aunque Rueda (2005) propone una ubicación asociada a la presencia de una zona de "pull apart" generada por fallas transcurrentes de desplazamiento lateral.

\section{METODOLOGÍA}

Para la caracterización sedimentológica y composicional, 27 muestras fueron recolectadas de las paredes internas del cráter del volcán a partir del levantamiento de una columna compuesta en el sector suroriental. Estas muestras fueron tratadas en los laboratorios del Instituto de Investigaciones en Estratigrafía (IIES) de la Universidad de Caldas. El tratamiento se llevó a cabo mediante el lavado de las muestras y posterior secado de las mismas a $60^{\circ} \mathrm{C}$. Posteriormente se realizó un tamizado diferenciando tamaños de 125,150 y $250 \mu \mathrm{m}$. Finalmente se realizó un análisis de componentes con lupa binocular en los tamaños $>250 \mu \mathrm{m}$ con un gramaje de muestra de 0,5 aproximadamente.

Para la caracterización morfológica, se realizó un análisis de fotografías aéreas el cual consistió en la determinación de las características morfológicas tanto del edificio volcánico como del cráter del VCM, teniendo en cuenta parámetros como tono, color, relieve, patrón de drenaje. 


\section{RESULTADOS}

\section{Estratigrafía}

\section{Características sedimentológicas}

Se realizó el levantamiento de una sección estratigráfica (FIGURA 2) de aproximadamente $13 \mathrm{~m}$ en la pared interna del cráter del VCM, en el sector suroriental del mismo $\left(4^{\circ} 29^{\prime} 17,5^{\prime \prime} \mathrm{N} 75^{\circ} 22^{\prime} 38,8^{\prime \prime} \mathrm{O}\right)$. A partir de la descripción detallada llevada a cabo en campo, se elaboró un esquema de la columna estratigráfica utilizando cinco litofacies definidas con base en el tamaño de grano, texturas y estructuras sedimentarias, color, selección y empaquetamiento; estas litofacies se repiten a lo largo de la columna (TABLA 1).
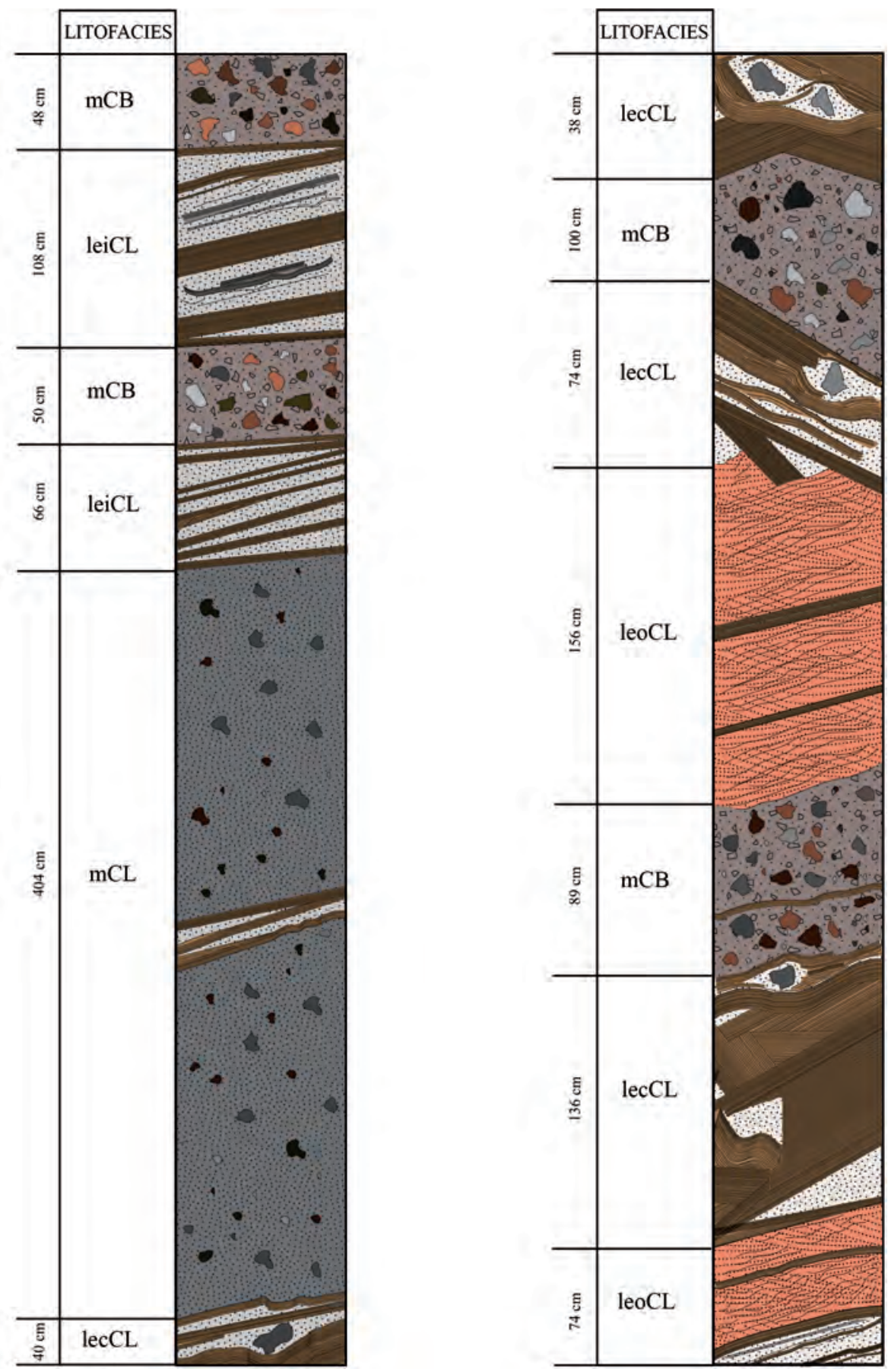

FIGURA 2. Columna estratigráfica del cráter del VCM, sector sur-oriental. 


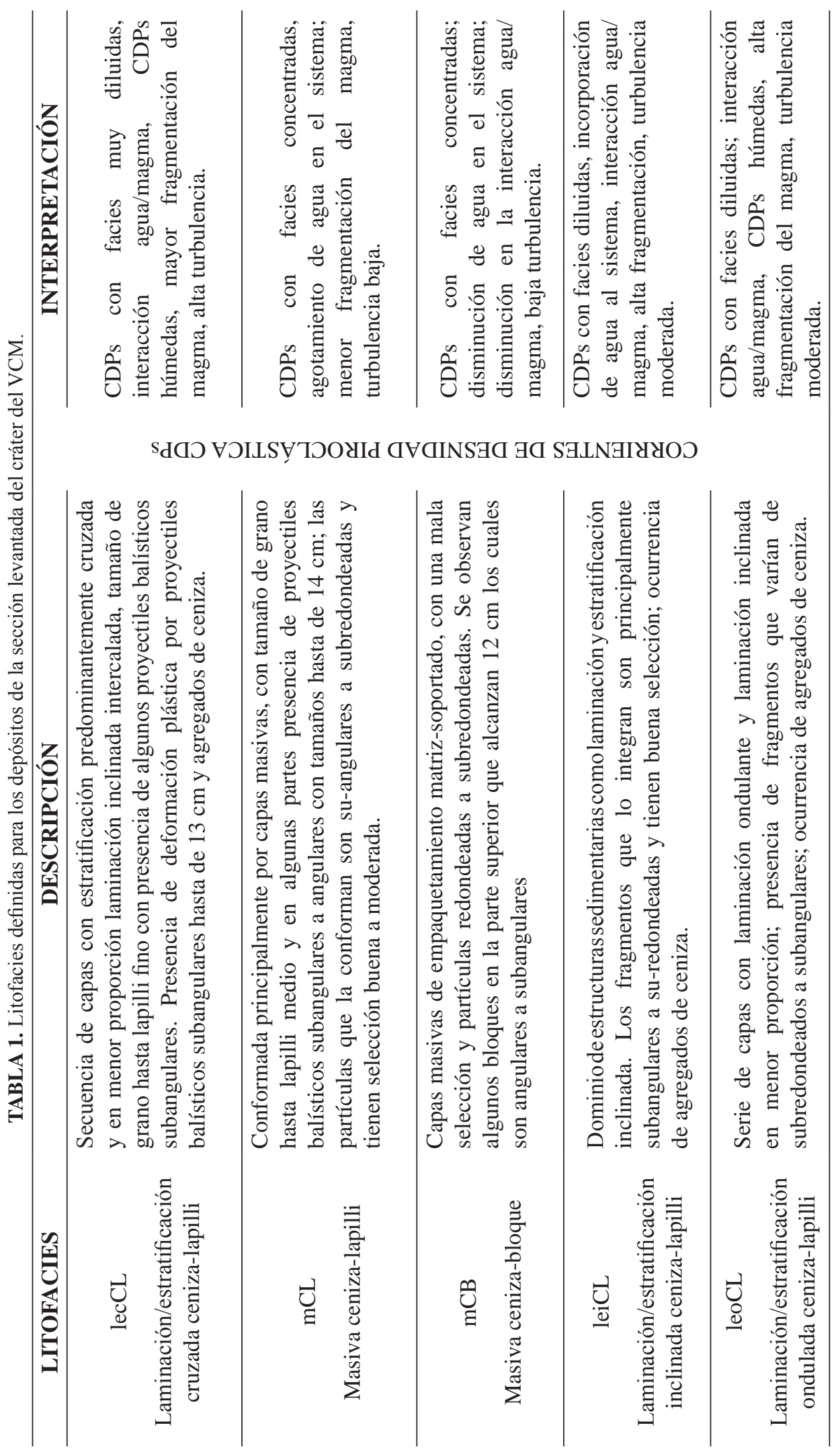


Dentro de las estructuras sedimentarias presentes, se destacan características tales como la intensa laminación inclinada, cruzada y eventualmente ondulada con espesores que varían de centimétricos a milimétricos (FIGURA 3A). Se destaca la presencia de agregados de ceniza tipo lapilli acrecional (FIGURA 3B) (cf. Scolamacchia et al., 2005) y de deformación tanto plástica como frágil asociada con impactos de proyectiles balísticos (FIGURA 3C y 3D).
También son características capas desde finas hasta gruesas (cf. Ingram, 1954) con estructura masiva y con empaquetamiento matriz-soportado (FIGURA 3E). En términos generales, se observa que los fragmentos son de subredondeados a angulares ( $c f$. Wadell, 1933) y tienen tamaños que varían desde de ceniza extremadamente fina hasta bloques $(<12 \mathrm{~cm}$; Wentworth y William, 1932; modificado por Murcia et al., 2013).

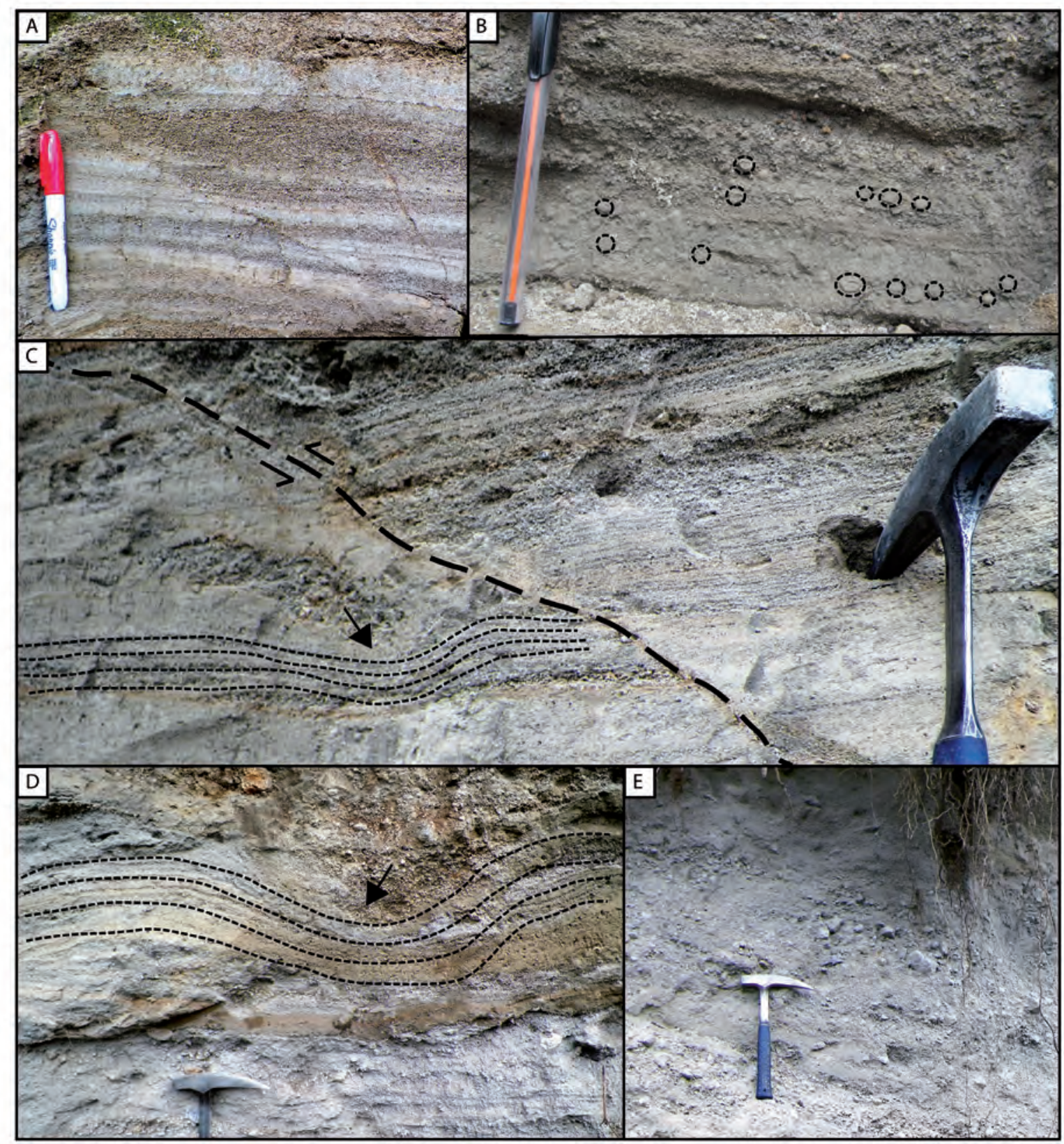

FIGURA 3. A. Litofacies leiCL. Nótese la laminación inclinada de bajo ángulo. B. Agregados de ceniza tipo lapilli acrecional la cual es característica de la litofacies leiCL. C. Litofacies lecCL. Nótese la deformación plástica por impacto de proyectil balístico y desplazamiento inverso sinsedimentario de las capas. D. Litofacies lecLC y deformación plástica por impacto de proyectil balístico. E. Litofacies mCB; una capa masiva con empaquetamiento matriz-soportado. 


\section{Análisis de componentes}

Con base en el análisis de los componentes bajo la lupa binocular, fue posible definir el tipo de fragmentos que conforman los depósitos. Estos corresponden a: líticos volcánicos (FIGURA 4A), vidrio vesiculado (i.e. pómez), cristales libres (cuarzo, plagioclasa, biotita, anfíbol, piroxeno; FIGURA 4B, 4C, 4D, 4E, 4F) y fragmentos metamórficos (FIGURA 4G). Así, se determinó que tanto los líticos volcánicos como el vidrio vesiculado corresponden a fragmentos juveniles y/o accesorios, mientras que los fragmentos metamórficos corresponden a fragmentos accidentales ( $c f$. Murcia et al., 2013). Los cristales, por su composición, también son considerados juveniles y/o accesorios teniendo en cuenta que la roca constituyente del basamento metamórfico no presenta este tipo de cristales.
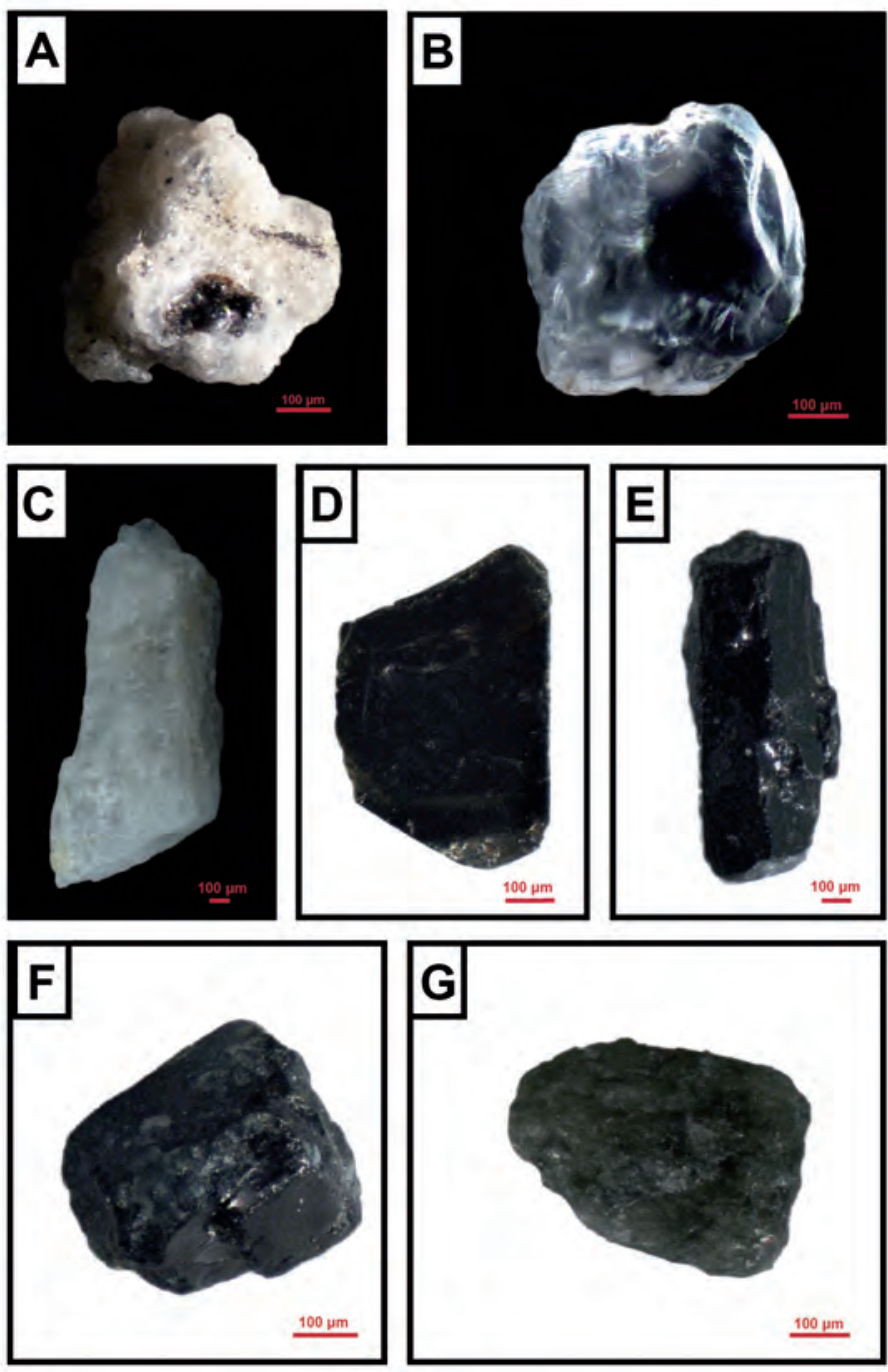

FIGURA 4. Fotografías de los componentes definidos para la sección levantada del cráter del VCM. A. Lítico volcánico. B. Cuarzo. C. Plagioclasa; D. Biotita. E. Anfíbol. F. Piroxeno. G. Lítico metamórfico.

En términos generales, es posible observar en toda la secuencia descrita, que los fragmentos volcánicos y cristales libres (principalmente de cuarzo) son dominantes sobre los fragmentos accidentales
(FIGURA 5). No obstante, se observa de base a techo de la secuencia un leve incremento en la cantidad de componentes accidentales involucrados. 

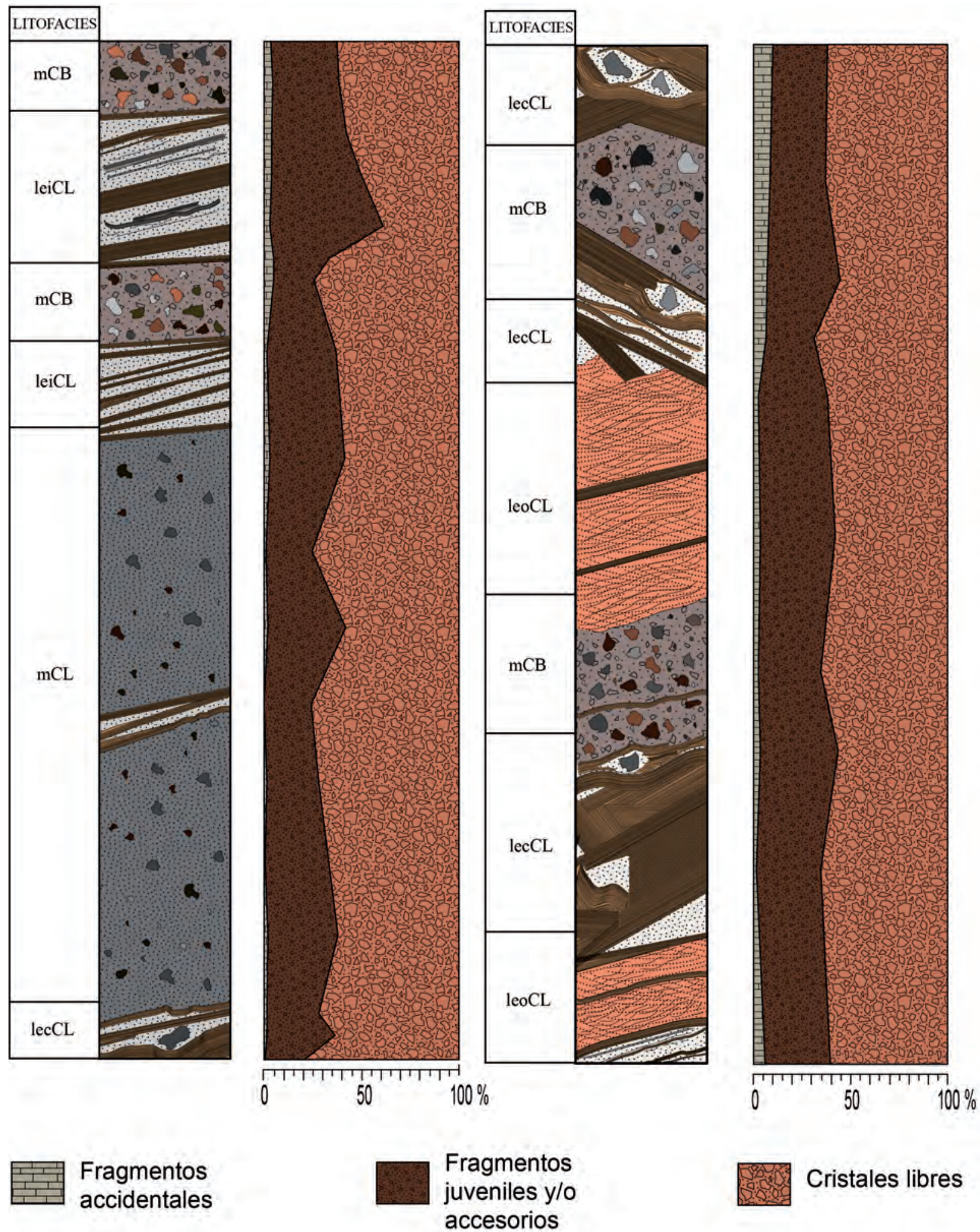

Cristales libres

FIGURA 5. Representación de la columna estratigráfica y diagramas de variación de componentes. 


\section{Morfología}

\section{Geomorfología}

La base y cima (incluyendo el domo) del VCM tienen una altura de 1524 y 2750 msnm, respectivamente. La base del edificio volcánico se estableció en el lugar donde cambia la topografía abruptamente y que en su mayoría corresponde a los cauces de los ríos que rodean el volcán (FIGURA 6A). El diámetro del edificio es de 6,6 km en su eje mayor (FIGURA 6A). El cráter tiene una altura de $2295 \mathrm{msnm}$ con un diámetro de 2,4 km (FIGURA 6A) y se encuentra destruido en la parte sur- occidental (FIGURA 6B). Internamente, se encuentra un domo de lava con abundante vegetación (FIGURA 6C) que tiene un diámetro basal máximo de $\sim 2,2 \mathrm{~km}$ y una altura máxima de $\sim 490 \mathrm{~m}$. El mapa de pendientes evidencia valores de planicies con inclinación de $0,1^{\circ}$ hasta laderas abruptas con ángulos de $67^{\circ}$ (FIGURA 6A). Asimismo, se observan valles profundos modelados por las corrientes de agua que a su vez rodean el edifico volcánico (FIGURA 6B). Hacia la parte nororiental y suroccidental del cráter se encuentra una morfología de montículos de corta extensión asociados al colapso de parte del domo (FIGURA 6D).

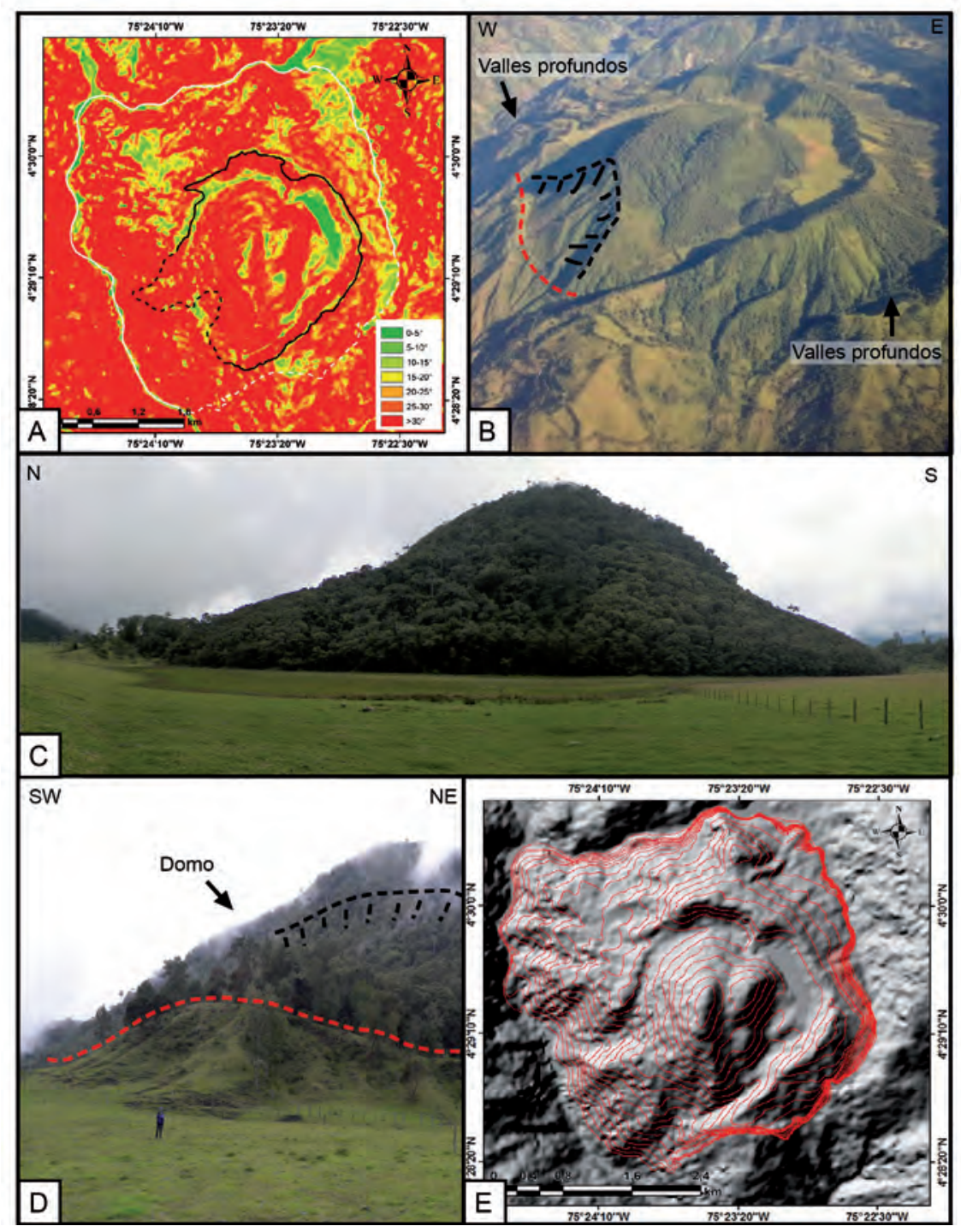

FIGURA 6. A. Mapa de pendientes del VCM, Línea blanca: delimitación de la base el edificio volcánico. Línea negra: delimitación del borde del cráter. B. Fotografía del VCM. Línea roja punteada: abertura del cráter hacia el SW. Línea negra punteada: cicatriz del domo. C. Domo intracratérico; nótese la abundante vegetación en el mismo. D. Representación fotográfica de algunas estructuras evidentes del VCM. Línea roja punteada: montículo encontrado en la base del cráter. Línea negra punteada: corona generada en el domo intracratérico. E. Mapa de contornos cada $50 \mathrm{~m}$ del VCM para establecer los parámetros morfométricos como lo son el índice de elipticidad e irregularidad. 


\section{Morfometría}

La morfometría del VCM fue cuantificada mediante la delimitación manual del edificio volcánico usando un DEM; de esta manera se obtuvieron diferentes parámetros morfométricos, así como las relaciones altura/ancho de la base $\left(H / W_{B}\right)$ y ancho de la cima/ ancho de la base $\left(W_{S} / W_{B}\right.$; e.g. Grosse et al., 2009; 2012; 2014).

Para la cuantificación, se elaboró un mapa de contornos con intervalos de $50 \mathrm{~m}$ para calcular los índices de elipticidad (ei) e irregularidad (ii; FIGURA 6E); esto permitió obtener valores cercanos a los reales. Dichos índices se calcularon para cada intervalo con el fin de obtener un promedio para todo el edificio volcánico. A partir de estas metodologías se obtuvieron los parámetros morfométricos como se muestran en la TABLA 2.

TABLA 2. Parámetros morfométricos determinados para el VCM.

\begin{tabular}{ll}
\hline \multicolumn{1}{c}{ Parámetros } & Valores \\
\hline Altura basal (msnm) & 1525 \\
Altura del cráter $(\mathrm{msnm})$ & 2295 \\
Altura del cono $\left(H_{C O}\right)$ & 770 \\
Diámetro basal $W_{C O}(\mathrm{~m})$ & 4564 \\
Diámetro del cráter $(\mathrm{m})$ & 2410 \\
Profundidad máxima del cráter (Dcr) & 170 \\
Radio basal $R b(\mathrm{~m})$ & 2282 \\
Radio del cráter $R s(\mathrm{~m})$ & 1205 \\
Volumen $\left(\mathrm{km}{ }^{3}\right)$ & 7 \\
Pendiente máxima & $67,35^{\circ}$ \\
Pendiente mínima & $0,098^{\circ}$ \\
Índice $H_{C O} / W_{C O}$ & 0,17 \\
Índice $W_{S} / W_{B}$ & 0,52 \\
Índice de elipticidad & 1,40 \\
Índice de irregularidad & 1,30 \\
\hline
\end{tabular}

\section{DISCUSIÓN}

\section{Estratigrafía}

\section{Depósitos volcaniclásticos y estilo eruptivo}

A lo largo de $13 \mathrm{~m}$ de la columna estratigráfica levantada en el cráter del VCM, se observaron una secuencia de depósitos volcaniclásticos en los cuales dominan las estructuras sedimentarias como laminación y estratificación cruzada, laminación inclinada y laminación ondulada. El tamaño de grano predominante de estas unidades varía entre ceniza extremadamente fina y lapilli fino, ocasionalmente con fragmentos tamaño bloque (Murcia et al., 2013). Estas características, así como el tamaño fino dominante en los depósitos y la buena selección de las partículas, indican que los depósitos fueron formados por CDPs diluidas (i.e. oleadas piroclásticas) (cf. Fisher, 1984; Branney y Kokelaar, 2002; Cano-Cruz y CarrascoNúñez, 2008; Brown y Andrews, 2015). Características como lapilli acrecional y deformación plástica por impacto de proyectil balístico, indican que las oleadas se depositaron a menos de $100^{\circ} \mathrm{C}$ (i.e. fueron oleadas húmedas; cf. Branney y Kokelaar, 2002), aunque la deformación frágil indica que oleadas secas también hicieron parte de la erupción. Estas características sugieren que las CDPs diluidas que conforman el cráter del VCM, estuvieron dominadas por alta turbulencia y baja densidad, y que posiblemente, hubo agua involucrada en la generación de varias de ellas. En conclusión, las características sedimentológicas en la secuencia levantada evidencian que en la construcción del cráter del VCM hubo variaciones en la dinámica eruptiva y que posiblemente la interacción agua/ magma jugó un papel significativo en tal construcción.

\section{Vesicularidad en los fragmentos juveniles $y / o$ accesorios}

En los depósitos de CDPs diluidas formados por la interacción agua/magma, la proporción de vidrio vesiculado (pómez) es muy escasa (Houghton y Wilson, 1989). En el caso del VCM, la poca cantidad de pómez $(<4 \%)$ evidencia que el magma no alcanzó a desarrollar alta vesicularidad antes de fragmentarse (cf. Gardner et al., 1996), lo que sugiere que ésta fue probablemente interrumpida durante el ascenso del magma, y que esto pudo haber sido causado por el encuentro del magma con un agente externo como un cuerpo de agua (Houghton y Wilson, 1989; Hiroi y Miyamoto, 2016). De esta manera, es posible sugerir que la erupción formadora del cráter actual de VCM estuvo fuertemente influenciada por la interacción agua/magma, proceso que impidió que el alto contenido de $\mathrm{H}_{2} \mathrm{O}$ en el magma $(>5,7$ wt. $\%$ si se consideran los valores reportados por Laeger et al., 2013) además de los otros volátiles, desarrollaran los procesos esperados de exsolución, nucleación, vesiculación y coalescencia (e.g. Cashman et al., 2000) y por ende, alta vesiculación en los fragmentos representativos del magma. 


\section{Fragmentos accidentales}

A lo largo de la columna estratigráfica levantada en el VCM se observaron fragmentos líticos accidentales en diversas cantidades. La aparición y proporción de dichos fragmentos, los cuales perteneces al Complejo Cajamarca, indican que, en el desarrollo de la erupción, las rocas del basamento fueron involucradas. Este proceso, ha sido asociado a un rompimiento térmicohidráulico de la pared de la roca cuando el agua entra en contacto con el magma ascendente (Büttner y Zimanowski, 1998). Así, el tipo y abundancia de fragmentos líticos encontrados en una secuencia estratigráfica en un volcán, pueden indicar el nivel de interacción agua/magma (Wohletz, 1986; Cano-Cruz y Carrasco-Núñez, 2008; Pardo et al., 2008; AgustínFlores et al., 2014; Zimanowski et al., 2015).

Para el caso del VCM, las características geológicas reportadas para esta región, indican que el basamento metamórfico posiblemente almacena agua subterránea en acuíferos confinados. Dichas rocas presentan esquistosidad además de estar afectadas por fracturamiento y cizallamiento, lo que contribuye a que las rocas presenten deformación y que contengan zonas de debilidad por donde el agua puede migrar y almacenarse. Así, los líticos metamórficos, sugieren una interacción agua/magma en algún lugar antes de que el magma alcanzara superficie. Adicionalmente, la baja cantidad de líticos accidentales sugiere que tal interacción fue relativamente baja asociada seguramente a la poca cantidad de agua disponible relacionada con el tipo de acuífero. Finalmente, aunque con base en las características composicionales encontradas no es posible sugerir una profundidad de fragmentación para el VCM, vale la pena mencionar que éstas ocurren a profundidades menores a $2 \mathrm{~km} \mathrm{y}$ que dependen de las condiciones de presión litostática principalmente (Valentine et al., 2014).

\section{Edad del cráter del VCM}

En las paredes internas del cráter del VCM se observaron fragmentos tamaño bloque $(>12 \mathrm{~cm})$ de redondez angular a subangular, así como fragmentos claramente balísticos con características similares; estos fragmentos podrían estar relacionados con la Unidad El Anillo (900 años) definida como la última erupción del VCM (Rueda, 2005), y la cual fue interpretada como formada por una erupción vulcaniana debido a los depósitos de flujo piroclástico de bloques y ceniza relacionados (ver también Murcia et al., 2010). No obstante, vale la pena mencionar que en esta unidad no fueron definidos los depósitos de CDPs como formadores de las paredes internas del cráter. Así, se propone que: 1) los depósitos aquí descritos corresponden a una fase avanzada de la erupción inicialmente vulcaniana que formó la Unidad El Anillo; 2) la presencia de proyectiles balísticos en algunas capas de la secuencia, sugieren que en algunos momentos de la erupción se involucraron fragmentos de los remanentes de un domo destruido al inicio de ésta.

\section{Formación del cráter actual del VCM}

La formación del cráter actual del VCM (FIGURA 7) está caracterizada por sucesivas fases eruptivas representadas en cinco litofacies. A la base, la aparición de la litofacies lecCL (FIGURA 2) sugiere una inyección de magma que interactuó cercano a la superficie con agua subterránea proveniente de un acuífero confinado asociado al basamento (Complejo Cajamarca). Así, se formaron CDPs diluidas con fragmentos juveniles (magma fragmentado) y/o accesorios (domo o conducto). Estas corrientes fueron seguramente húmedas $\left(<100^{\circ} \mathrm{C}\right)$, considerando la presencia de agregados de ceniza (lapilli acrecional) así como de proyectiles balísticos que deformaron plásticamente (i.e. deformación dúctil) las capas. Igualmente, la abundancia de estructuras sedimentarias como laminación inclinada y cruzada sugieren alta turbulencia asociada con una muy baja concentración de fragmentos en los flujos. Composicionalmente, la aparición de esta litofacies refleja una escasa presencia de fragmentos accidentales (1,17\%; i.e. basamento), lo que indica una baja disrupción del basamento durante la erupción.

Suprayaciendo, la ocurrencia de la litofacies mCL (FIGURA 2) sugiere la formación de CDPs más concentradas (menor turbulencia) con respecto a la litofacies lecCL, teniendo en cuenta que exhiben una estructura masiva. Su formación puede estar asociada al agotamiento temporal de agua en el sistema, lo que ocasionó la generación de flujos con mayor cantidad y tamaño de fragmentos, a su vez asociados con una fragmentación más gruesa del magma o fragmentación el domo. Durante su emplazamiento, la depositación fue afectada por proyectiles balísticos como evidencia de fragmentos del domo aún presentes parcialmente dentro del cráter. La cantidad de fragmentos accidentales en esta litofacies $(1,17 \%)$ indica que la disrupción del basamento continuó siendo leve y que los flujos siguieron siendo dominados por magma fragmentado y/o fragmentos del domo o el conducto. 


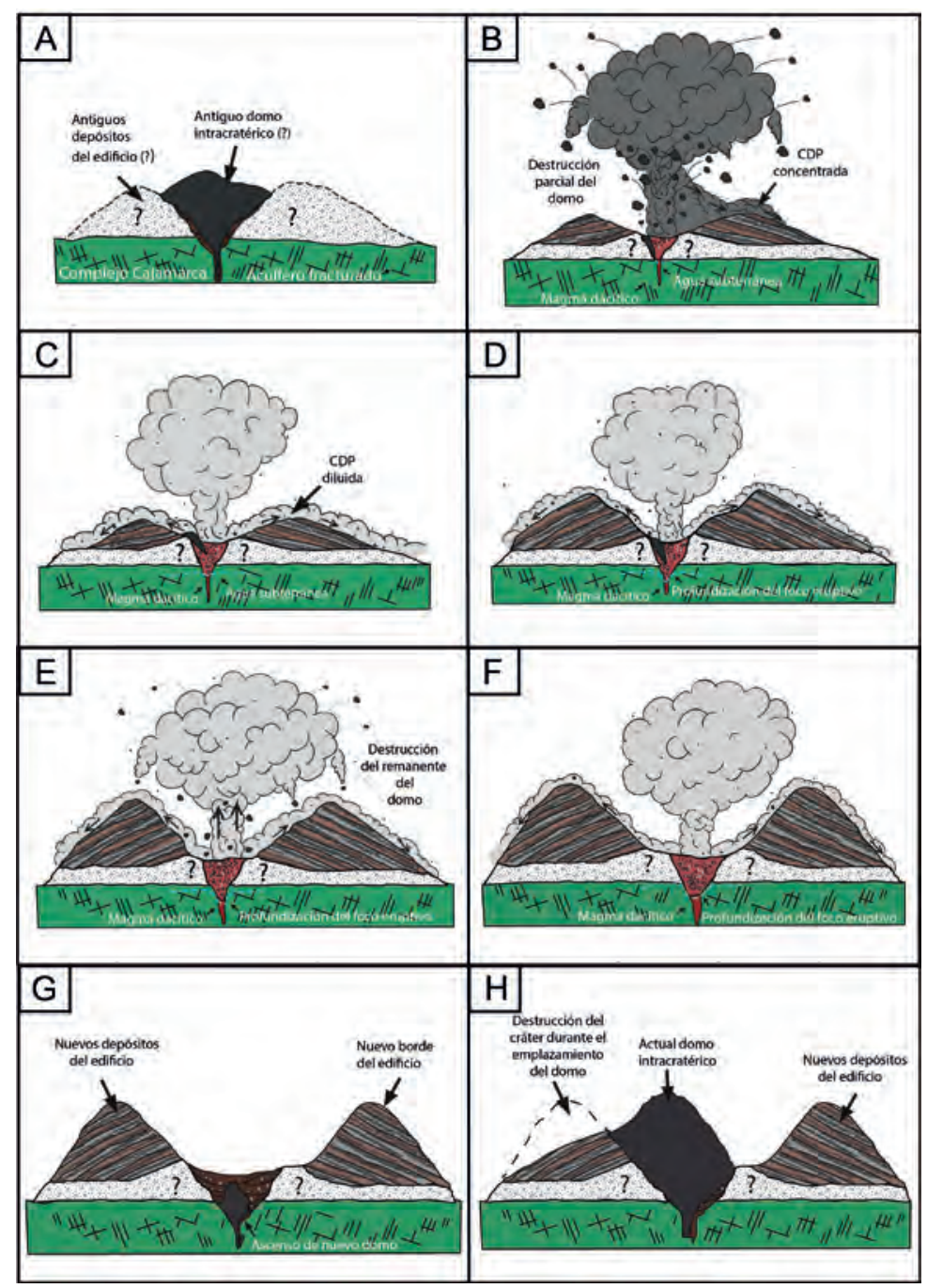

FIGURA 7. Representación esquemática de la formación del cráter actual del VCM. A. Representa el estado inicial del volcán, taponado por un domo. B. Representa un estado avanzado de la erupción, en donde después de generarse los depósitos de flujos piroclásticos de bloques y ceniza pertenecientes a la Unidad El Anillo se generaron varias CDPs como las reportadas en este trabajo. C. a H. Representan la secuencia eruptiva como se describe en el texto.

La aparición de la litofacies leiCL (FIGURA 2) sugiere nuevamente un aumento en la turbulencia de los flujos que la generaron, teniendo en cuenta la formación de laminación inclinada sin la presencia de otras estructuras características de mayor turbulencia. Su formación está relacionada con la incorporación de agua al sistema que ocasionó nuevamente su interacción con el magma; en esta litofacies se da la ocurrencia de agregados de ceniza (lapilli acrecional) lo cual indica que las CDPs fueron húmedas.

La ocurrencia de la litofacies mCB (FIGURA 2) evidencia la formación de CDP altamente concentrada, considerando la estructura masiva de los depósitos. Esta litofacies parece responder a una nueva disminución en la interacción de agua con el magma.

De nuevo, la presencia de la litofacies leiCL (FIGURA 2) sugiere la formación de CDPs diluidas indicando una mayor turbulencia en los flujos con relación a la litofacies infrayacente, teniendo en cuenta la presencia de diferentes estructuras como laminación inclinada y laminación ondulada. Su formación puede estar asociada a la nueva incorporación de agua en el sistema.

De nuevo, la presencia de la litofacies mCB (FIGURA 2) siguiere la formación de una CDP con facies más 
concentradas que la unidad anterior, teniendo en cuenta una estructura masiva y empaquetamiento matriz-soportado así como presencia de deformación frágil por impacto de proyectil balístico. Esta litofacies parece reflejar una nueva disminución de agua en el sistema y una nueva destrucción de los remanentes del domo aún existentes.

La aparición de la litofacies leoCL (FIGURA 2) indica la formación de CDPs húmedas $\left(<100^{\circ} \mathrm{C}\right)$ como lo evidencia la presencia de lapilli acrecional y una turbulencia moderada reflejada por la presencia de laminación ondulada. La nueva aparición de la litofacies leiCL (FIGURA 2) indica alta turbulencia en los flujos que las generaron con base en la presencia de abundante laminación cruzada y humedad en las CDPs indicada por la deformación plástica por impacto de proyectil balístico. Ambas litofacies reflejan también la influencia de agua en la fragmentación del magma.

La nueva presencia de la litofacies mCB (FIGURA 2) sugiere la formación de una CDP con facies concentrada; los depósitos carecen de estructuras y presentan deformación frágil, lo que a su vez sugiere una disminución de agua en el sistema y por ende menos energía (fragmentación y turbulencia) involucrada.

Un espeso nivel en la secuencia, otra vez con la presencia de la litofacies leoCL (FIGURA 2) sugiere la formación de CDPs diluidas como lo evidencia la laminación ondulada e inclinada. Estas características sugieren un aumento en la interacción de agua y magma.

El techo de la secuencia corresponde a la aparición intercalada entre las litofacies lecCL, mCB y de nuevo lecCL (FIGURA 2); la litofacies lecCL evidencia un buen desarrollo de estructuras sedimentarias que indican alta turbulencia (laminación cruzada) así como la presencia de deformación dúctil en las capas por impacto de proyectil balístico, mientras que la litofacies $\mathrm{mCB}$ refleja una $\mathrm{CDP}$ con facies más concentrada y a su vez menor turbulencia; esta litofacies responde también a una disminución de agua en el sistema. Este sector de la secuencia presenta una mayor cantidad de fragmentos accidentales (7-10\%), lo cual sugiere que el proceso de fragmentación continuó involucrando el basamento hasta el final de la erupción.

\section{Morfología}

\section{Rasgos geomorfológicos}

Para el VCM, fue posible determinar diferentes procesos involucrados en su evolución, los cuales corresponden principalmente a procesos eruptivos que formaron el edificio volcánico y que fueron básicamente explosivos (cuatro erupciones plinianas/ subplinianas y dos vulcanianas; Rueda, 2005). Los procesos constructivos del edificio volcánico asociados a las diferentes erupciones a través del tiempo, tienen un control dominante respecto a la relación con los procesos erosivos de la zona tropical, los cuales se asocian con la dinámica climática, fluvial, eólica y gravitacional ( $c f$. Dóniz-Páez y Coello de la Plaza, 2004; Alcalá-Reygosa et al., 2016). El área del VCM corresponde a clima templado a cálido, con una temperatura media de $20^{\circ} \mathrm{C}$, una precipitación pluviométrica anual de $1150 \mathrm{~mm}$, y una humedad relativa promedio del $85 \%$; las épocas secas y lluviosas fluctúan comúnmente en el transcurso del año. Se trata de una región montañosa que se caracteriza por tener relieves moderados a abruptos, cuya vegetación, según la clasificación de Holdridge (Espinal, 1977), va desde un bosque muy húmedo premontano (bmh $\mathrm{PM}$ ) hasta bosque muy húmedo montano Bajo (bmh - MB) (www.sgc.gov.co). En conclusión, aunque el edificio volcánico actual del VCM refleja una buena conservación, la magnitud de las sucesivas erupciones sugiere procesos constructivos y destructivos del edificio volcánico. En menor proporción, los patrones erosivos han modelado el edificio volcánico actual. Así, la relación de conservación del edificio entre las diferentes erupciones es difícil de establecer.

\section{Destrucción del cráter}

Los procesos denudativos en el VCM son evidenciados mediante diferentes estructuras y características morfológicas; dichos aspectos pueden estar asociados a procesos sin-eruptivos o post-eruptivos en la evolución morfológica del edificio volcánico. La presencia de montículos que se encuentran en la base interna del cráter, sugiere un colapso del domo intracratérico el cual está relacionado con una cicatriz de deslizamiento (FIGURA 6B). Esta estructura evidencia un alto grado de alteración relacionado con procesos de actividad hidrotermal; en la cicatriz, se encuentran fuentes de gas. Este domo intracratérico es interpretado como la fase efusiva con la que terminó la última erupción del VCM (Laeger et al., 2013). Así, los montículos observados en la parte basal del cráter, si bien sugieren un proceso de denudación del domo, podrían haber ocurrido durante o mucho tiempo después de la erupción. De la misma manera, la abertura del cráter hacia el suroccidente es una morfología que evidencia un declive en la conservación del edificio volcánico. 
En conclusión, en el momento en que aún no se terminaba de formar el cráter actual, es posible que, en el proceso de construcción de éste, el domo estuviese ascendiendo en mayor proporción hacia ese sector lo cual afectó directamente al cráter, deformándolo y posteriormente destruyéndolo. Asimismo, procesos post-eruptivos asociados a erosión podrían haber afectado el domo sometiéndolo a una destrucción parcial y así, generando colapsos que a su vez contribuyeron con la destrucción del cráter en este sector (FIGURA 8).

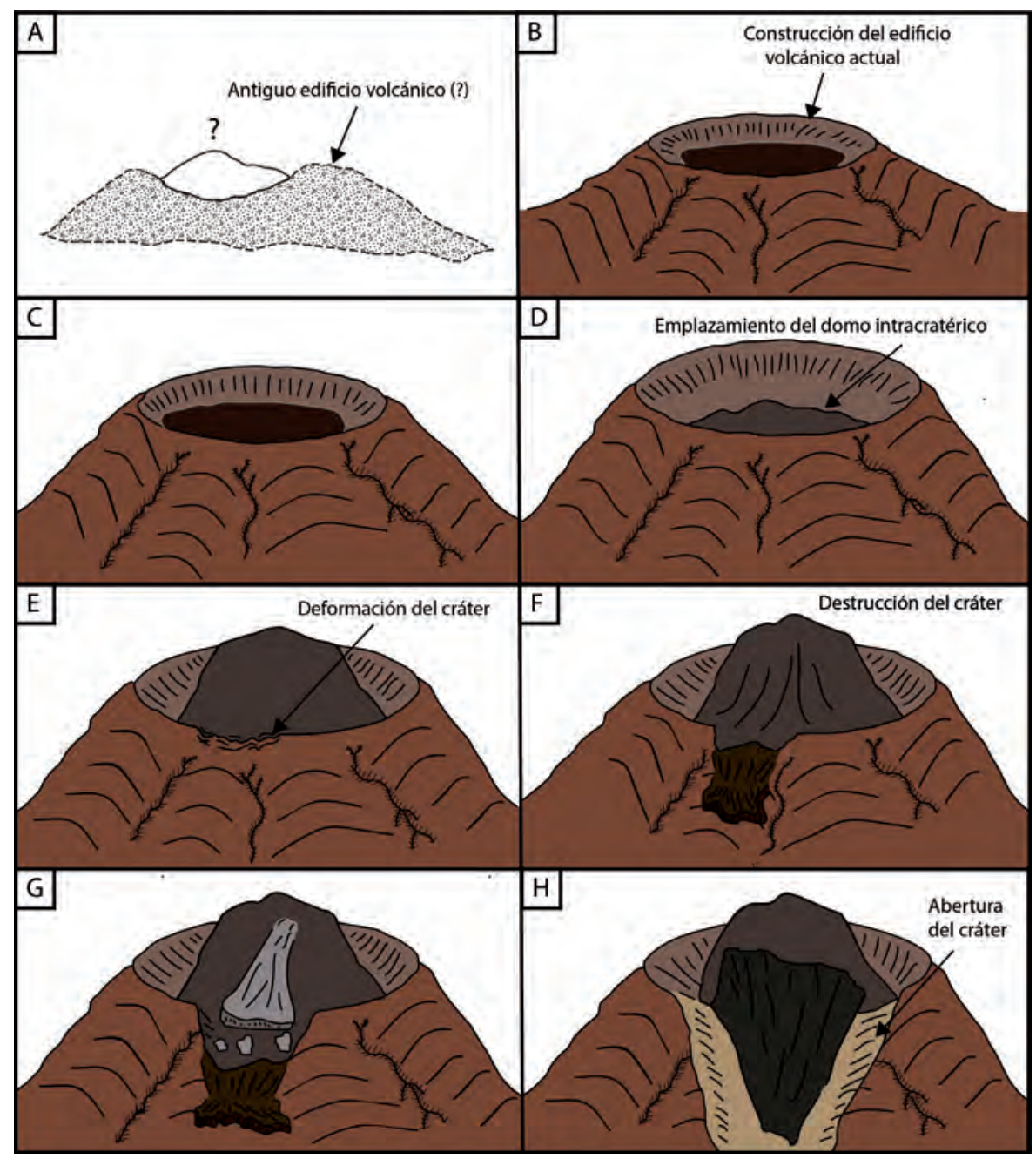

FIGURA 8. Modelamiento de proceso de destrucción del cráter actual del VCM. A. Representa el estado inicial del volcán, taponado por un domo. B. y C. Representa un estado de construcción de un nuevo cráter a medida que avanza la erupción. D. y E. Emplazamiento y paulatina deformación del cráter. F. a H. Proceso de destrucción del cráter actual de un domo intracratérico generando una abertura en el mismo.

\section{Índices morfométricos}

Según Grosse et al. (2012) parámetros morfométricos como el tamaño, la forma, la altura, el ancho, así como algunas relaciones cuantitativas han sido utilizadas para la interpretación en términos de construcción y destrucción de los edificios volcánicos; estos estudios se enfocan en tipos de volcanes específicos y en parámetros cuya elección depende del tipo de volcán o en los objetivos del estudio (Grosse et al., 2009; 2012; 2014).
Para la delimitación manual del edificio volcánico, se calculó un volumen de $7 \mathrm{~km}^{3}$ y un área de $13 \mathrm{~km}^{2}$. Este volumen es bastante grande comparándolo, por ejemplo, con el volcán Puracé $\left(V=0,1693 \mathrm{~km}^{3}\right)$ sin embargo, es un volcán con pequeño volumen comparándolo con volcanes compuestos como el Nevado del Tolima $\left(V=21 \mathrm{~km}^{3}\right)$ o el volcán Cerro Bravo $\left(V=10 \mathrm{~km}^{3}\right.$; Grosse et al., 2014. Es importante considerar que el VCM es una estructura reciente 
comparada con algunos de otros volcanes de la PVTSC y eso podría asociarse a su diferencia volumétrica; sin embargo, relacionándolo con una corta historia eruptiva y una altura menor respecto a estas otras estructuras, es un volumen considerable. El VCM evidencia un valor $e i=1,406$ y $i i=1,305$, lo cual sugiere una estructura heterogénea en forma y geometría con tendencia a tener elipticidad e irregularidad sin ser los valores muy altos (FIGURA 9A). La relación $H / W_{B}=0,168$ es una relación que sugiere una forma cónica ( $c f$. Grosse et al., 2009), sin embargo, la relación $W_{S} / W_{B}=0,528$ (cf. Grosse et al., 2009), es un valor superior que sugiere una forma maciza y además un valor poco común para un volcán compuesto (FIGURA 9B). Es importante mencionar que la conservación del VCM es menor a la de otros volcanes por el tipo de material que podría estar erosionándose, ya que las sucesivas capas de flujos de lava caracterizan los volcanes más cónicos y a su vez mejor conservados. El cráter Del VCM tiene una profundidad máxima de 170 m y un diámetro de 2,4 km con pendientes moderadas menores a las laderas externas de volcán; estos parámetros sugieren un mayor desarrollo del cráter relacionado con otros volcanes poligenéticos de la PVTSC (e.g. volcán Cerro Bravo y el volcán Nevado del Tolima), y a su vez, relacionado al estilo eruptivo y características intrínsecas de la erupción.

Los parámetros morfométricos arrojados en el análisis del VCM no permiten hacer una clasificación con las condiciones que propone Grosse et al. (2009) ya que la relación $H / W_{B}$ correspondería a un cono y con la relación $W_{S} / W_{B}$ clasificaría como un macizo. Esto se vincula directamente con la morfología que desarrolla el volcán, ya que hay una estrecha relación entre el valor $W_{S} / W_{B}$ y el desarrollo del cráter. Este último valor sugiere degradación del VCM, sin embargo, este valor se asocia más a la génesis del volcán, así como a las características de las erupciones y al desarrollo de un profundo y extenso cráter.

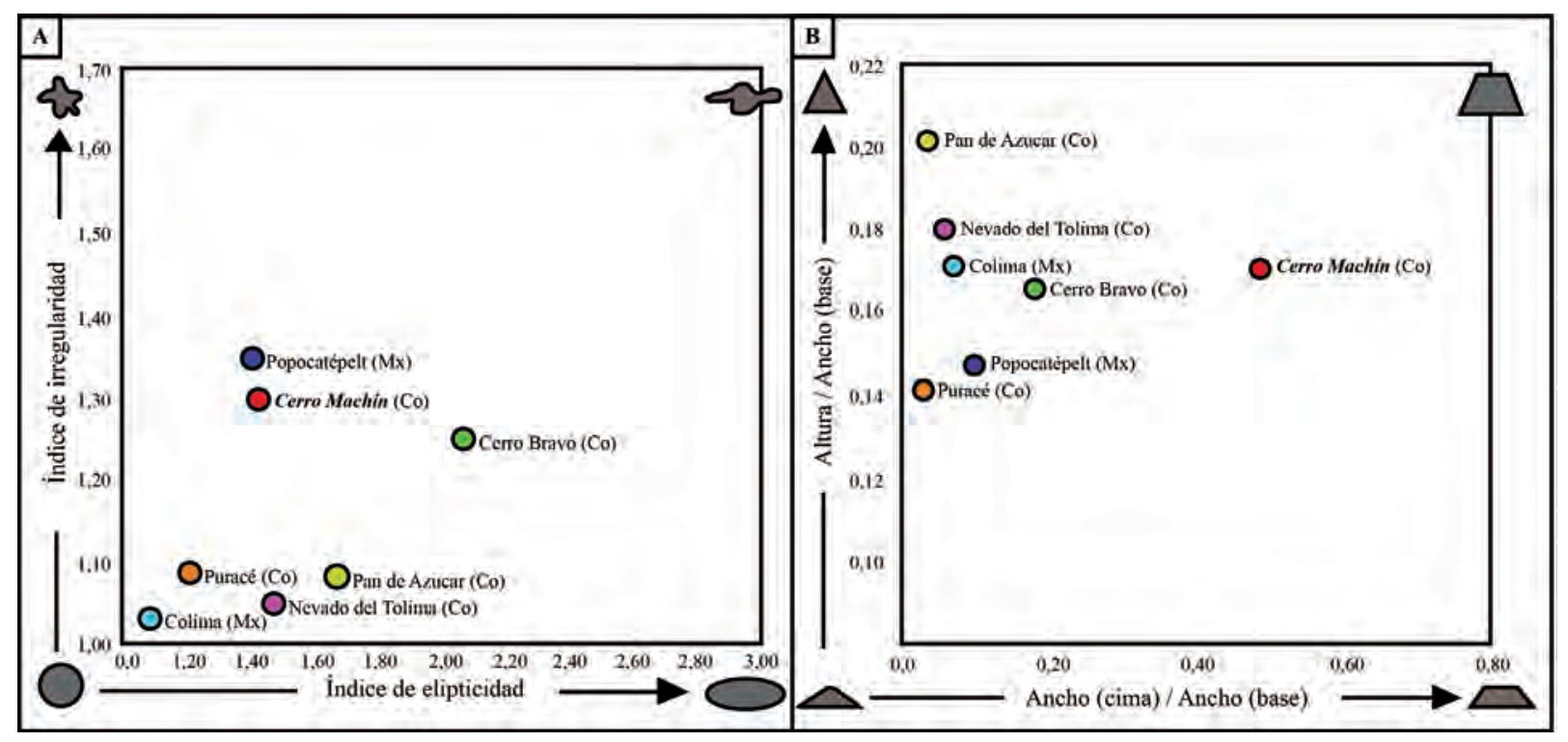

FIGURA 9. A. Gráfica de relación entre índice de elipticidad vs índice de irregularidad (adaptada de Grosse et al., 2012). B. Gráfica de relación entre $H / W_{B} W_{S} / W_{B}$ (adaptada de Grosse et al., 2012). Las siglas Co y Mx están asociadas a la ubicación geográfica de cada volcán y corresponden a los países de Colombia y México respectivamente.

\section{Clasificación de la estructura del VCM}

Cómo se ha mencionado a lo largo del trabajo, el VCM ha registrado sucesivas erupciones durante el Holoceno, lo cual es indicativo de vulcanismo poligenético. El VCM ha sido denominado "volcán compuesto" (cf. Murcia et al., 2010) siguiendo, por ejemplo, la clasificación de De Silva y Lindsay (2015) para este tipo de volcanes.
Con base en su morfología (pendientes $>10^{\circ}$ ), así como en los depósitos que forman su cráter (CDPs diluidas), el VCM también podría ser clasificado como un "cono de toba” (i.e. un volcán monogenético formado por actividad freato-magmática principalmente; $c f$. De Silva y Lindsay, 2015); de hecho, en diferentes trabajos (e.g. Méndez, 2001; Cepeda et al., 1999a y 1999b; Rueda, 2000; Cortés, 2001a y 2001b; Obando y Ramos, 2003; Hurtado 
y Murcia, 2003; Rueda, 2005), éste ha sido nombrado con un término para definir estructuras monogenéticas. Así, el VCM ha sido definido como un anillo de toba (tuff ring) (i.e. pendientes $<10^{\circ}$; Murcia et al., 2010), o como un "anillo piroclástico" (Rueda, 2005), y actualmente, como "anillo piroclástico complejo" (www.sgc.gov.co). La evidencia morfológica, así como la estratigráfica (i.e. columna estratigráfica), y la ausencia de discordancias en la columna levantada en su cráter, responden a que éste fue construido posiblemente durante su última erupción (900 años AP), erupción que finaliza con una fase efusiva que se ve reflejada en el emplazamiento de un domo. Es importante considerar que la sección estratigráfica levantada corresponde a una porción de las paredes internas del cráter actual del VCM; asimismo, son depósitos que conforman la última erupción, ya que como se mencionó anteriormente hay más depósitos volcaniclásticos asociados a esta (depósitos piroclásticos de bloques y ceniza; Rueda, 2005).

En este orden de ideas, en este trabajo se plantea el interrogante del término correcto para nombrar el VCM. Así, se propone definir el VCM como un "volcán compuesto con carácterísticas de cono de toba”; esto con base en la historia eruptiva del volcán en primera instancia y, por otro lado, en la morfología y depósitos del cráter, típicos de un volcán monogenético.

\section{CONCLUSIONES}

Los depósitos volcaniclásticos presentes en el cráter del VCM, corresponden a depósitos de CDPs diluidas caracterizados por presentar múltiples estructuras sedimentarias (laminación y/o estratificación paralela, cruzada e inclinada, agregados de ceniza, gradación, huellas de impacto). La presencia de lapilli acrecional y deformación plástica por proyectil balístico en algunas capas de la secuencia indican que algunas corrientes se depositaron a temperaturas menores a $100^{\circ} \mathrm{C}$ (i.e. oleadas piroclásticas húmedas). La presencia de proyectiles balísticos sugiere la existencia de los remanentes de un domo el cual taponaba el conducto al momento del inicio de la erupción y que se fueron fragmentando paulatinamente a lo largo de la misma, es decir, el cráter estuvo parcialmente abierto durante gran parte de la erupción.

La ausencia de evidencias (e.g. paleosuelos) de un paso de tiempo importante entre los depósitos de toda la secuencia levantada en el cráter, así como sus características morfológicas, indican que éste se formó por actividad asociada a una sola erupción. La escasa y/o casi nula cantidad de vidrio vesiculado en los depósitos que conforman el cráter sugieren que la erupción que lo formó no corresponde a ninguna de las erupciones plinianas reportadas para la historia eruptiva del VCM; las características del cráter como se reportan en este trabajo, sugieren que el cráter fue formado en su última erupción (erupción vulcaniana ocurrida hace 900 años).

El cráter actual del VCM está caracterizado por haber sido formado a partir de sucesivas fases eruptivas como resultado de mecanismos de fragmentación de tipo magmático y freato-magmático. Asimismo, la presencia de fragmentos accidentales pertenecientes al basamento (Complejo Cajamarca) sugiere un mecanismo de fragmentación asociado con interacción agua/magma (freato-magmatismo). La presencia de agua en el sistema en el momento de la última erupción del VCM debe estar asociada a la existencia de un acuífero en el área.

La relación $\left(H_{C O} / W_{C O}\right)$ del cono refleja una denudación de las geoformas asociadas. Evidencia de los procesos de denudación son los montículos que se encuentran en la base interna del cráter provenientes del colapso del domo intracratérico. La relación $W_{S} W_{B}(\mathrm{R}=0,528)$ es un valor superior y además un valor poco común para un volcán compuesto; este valor está relacionado con las características de la erupción que formó el cráter del VCM.

La destrucción del cráter, representado por su abertura actual hacia el SW, está asociada al ascenso del domo como última fase de la última erupción.

Teniendo en cuenta las características arquitectónicas y sedimentológicas de los depósitos de oleadas piroclásticas analizados en este trabajo y la de los parámetros morfométricos, así como lo datos obtenidos de trabajos anteriores y registros históricos del VCM, es posible sugerir que este volcán si bien, es un volcán poligenético (volcán compuesto), por su morfología, depósitos formadores del cráter (CDPs) y pendientes $>10^{\circ}$, tiene características de volcán monogenético tipo "cono de toba".

\section{AGRADECIMIENTOS}

Al Instituto de Investigaciones en Estratigrafía (IIES) de la Universidad de Caldas por su apoyo para con el desarrollo de este proyecto. A los revisores Carlos Borrero y Lilia Arana por los acertados comentarios que ayudaron a hacer mucho más claro este manuscrito. 


\section{REFERENCIAS}

Agustín-Flores, J., Németh, K., Cronin, S.J., Lindsay, J.M., Kereszturi, G., Brand, B.D., and Smith, I.E. (2014). Phreatomagmatic eruptions through unconsolidated coastal plain sequences, Maungataketake, Auckland volcanic field (New Zealand). Journal of Volcanology and Geothermal Research, 276, 46-63. doi: 10.1016/j. jvolgeores.2014.02.021.

Alcalá-Reygosa, J., Palacios, D., and ZamoranoOrozco, J.J. (2016). Geomorphology of the Ampato volcanic complex (Southern Perú). Journal of Maps, 12, 1160-1169.

Arcila, M., Muñoz, A., y De Vicente, G. (2002). Análisis sismotectónico de la convergencia Caribe, Nazca y Sudamérica. I Simposio Colombiano de Sismología. INGEOMINAS, Bogotá.

Blanco-Quintero, I.F., García-Casco, A., Toro, L.M., Moreno, M., Ruiz, E.C., Vinasco, C.J., Cardona, A., Lázaro, C., and Morata, D. (2014). Late Jurassic terrane collision in the northwestern margin of Gondwana (Cajamarca complex, eastern flank of the Central Cordillera, Colombia). International Geology Review, 56, 1852-1872. doi: 10.1080/00206814.2014.963710.

Bourdon, E., Eissen, J.P., Gutscher, M.A., Monzier, M., Hall, M., and Cotton, J. (2003). Magmatic response to early aseismic ridge subduction: The Ecuadorian margin case (South America). Earth and Planetary Science Letters, 205(3-4), 123138. doi: 10.1016/S0012-821X(02)01024-5.

Branney, M.J., and Kokelaar, B.P. (2002). Pyroclastic density currents and the sedimentation of ignimbrites. London: Geological Society of London.

Brown, R.J., and Andrews, G.D.M. (2015). Deposits of pyroclastic density currents. In: H. Sigurdsson, B. Houghton, S.R. McNutt, H. Rymer, J. Stix (eds.). The Encyclopedia of Volcanoes (pp. 631-648). 2nd edition, Academic Press, Elsevier, USA.

Büttner, R., and Zimanowski, B. (1998). Physics of thermohydraulic explosions. Physical Review, 57(5), 5726-5729.
Cano-Cruz, M., y Carrasco-Núñez, G. (2008). Evolución de un cráter de explosión (maar) riolítico: Hoya de Estrada, campo volcánico Valle de Santiago, Guanajuato, México. Revista Mexicana de Ciencias Geológicas, 25(3), 549564.

Cashman, K., Sturtevant, B., Papale, P., and Navon, O. (2000). Magmatic fragmentation. In: H. Sigurdsson, B. Houghton, S.R. McNutt, H. Rymer (eds.). The Encyclopedia of Volcanoes (pp. 459471). 2nd edition, Academic, Elsevier, USA.

Cepeda, H., Cortés, G.P., Macías, J.L., Méndez, R.A., Monsalve, M.L., Rueda, H., y Siebe, C. (1999a). El Cerro Machín, un volcán holocénico explosivo en el centro de Colombia. IV Reunión Técnica de la Comisión de Geofísica, Departamento de Geodesia y Topografía - UNT, Tucumán Argentina.

Cepeda, H., Muñoz, F., y Velásquez, E. (1999b). El volcán Cerro Machín: La prevención de desastres y el ordenamiento territorial. Bogotá, INGEOMINAS.

Cepeda, H., Murcia, L.A., Monsalve, M.L., Méndez, R.A., y Núñez, A. (1995). Volcán Cerro Machín, Departamento del Tolima, Colombia: Pasado, Presente y Futuro. INGEOMINAS, Popayán. Informe Interno.

CHEC. (1983). Investigación geotérmica Macizo Volcánico del Ruíz. CHEC S.A. Informe Final, 127p.

Cortés, G.P. (2001a). Estudio geológico de los depósitos del Lahar asociados a la actividad eruptiva del Volcán Cerro Machín. Colombia INGEOMINAS, Informe Interno, inédito, 96p.

Cortés, G.P. (2001b). Lahares asociados a la actividad eruptiva del Volcán Cerro Machín. VIII Congreso Colombiano de Geología. Manizales, Colombia.

De Silva, S., and Lindsay, J.M. (2015). Primary volcanic landforms. In: H. Sigurdsson, B. Houghton, S.R. McNutt, H. Rymer, J. Stix (eds.). The encyclopedia of volcanoes (pp. 273-297). 2nd edition, Lodon: Academic Press, Elsevier. 
Diederix, H., Audemard, F., Osorio, J.A., Montes, N., Velandia, F., y Romero, J. (2006). Modelado morfotectónico de la falla transcurrente de Ibagué, Colombia. Revista de la Asociación Geológica Argentina, 61(4), 492-503.

Dóniz-Páez, F.J. (2005). Los campos volcánicos basálticos monogénicos de la isla de Tenerife (Canarias, España). Estudios Geográficos, 66(259), 461-480.

Dóniz-Páez, F.J., y Coello de la Plaza, J.E. (2004). Evolución geomorfológica del conjunto volcánico subhistórico de Montaña Cascajo (Dorsal de Bilma-Abeque) en Tenerife. Investigaciones Geográficas, 35, 151-164. doi: 10.14198/ INGEO2004.35.02.

Espinal, L.S. (1977). Formaciones vegetales de Colombia. Memoria explicativa sobre el mapa ecológico. Instituto Geográfico Agustín Codazzi. Bogotá. 238p.

Fisher, R.V. (1984). Submarine volcaniclastic rocks. In: B.P. Kokelaar and M.F. Howells (ed.). Marginal basin geology: Volcanic and associated sedimentary and tectonic processes in modern and ancient arginal basins (pp. 5-27). London: Geological Society, Special Publications.

Fisher, R.V., and Schmincke, H.U. (1984). Pyroclastic rocks. Berlín: Springer Verlag.

Gardner, J.E., Thomas, R.M., Jaupart, C., and Tait, S. (1996). Fragmentation of magma during Plinian volcanic eruptions. Bulletin of Volcanology, 58(23), 144-162. doi: 10.1007/s004450050132.

Grosse, P., Euillades, P.A., Euillades, L.D., and van Wyk de Vries, B. (2014). A global database of composite volcano morphometry. Bulletin of Volcanology, 76, 784-800. doi: 10.1007/s00445013-0784-4.

Grosse, P., van Wyk de Vries, B., Euillades, P.A., Kervyn, M., and Petrinovic, I.A. (2012). Systematic morphometric characterization of volcanic edifices using digital elevation models. Geomorphology, 136(1), 114-131. doi: 10.1016/j. geomorph.2011.06.001.

Grosse, P., van Wyk de Vries, B., Petrinovic, I.A., Euillades, P.A., and Alvarado, G. (2009).
Morphometry and evolution of arc volcanoes. Geology, 37(7), 651-654. doi: 10.1130/ G25734A.1.

Hiroi, Y., and Miyamoto, T. (2016). Relationship between eruptive style and vesicularity of juvenile clasts during eruptive episode $\mathrm{A}$ of Towada Volcano, Northeast Japan. Journal of Volcanology and Geothermal Research, 325, 8697. doi: 10.1016/j.jvolgeores.2016.06.009.

Houghton, B.F., and Wilson, C.J.N. (1989). A vesicularity index for pyroclastic deposits. Bulletin of Volcanology, 51(6), 451-462. doi: 10.1007/BF01078811.

Hurtado, B., y Murcia, H. (2003). Caracterización del depósito de flujo de escombros de Chicoral, volcán Cerro Machín, Colombia. Tesis, Universidad de Caldas, Manizales, Colombia.

Ingram, R.L. (1954). Terminology for the thickness of stratification and parting units in sedimentary rocks. GSA Bulletin, 65(9), 937-938. doi: 10.1130/0016-7606(1954)65[937:TFTTOS]2.0. CO;2.

Karátson, D., Favalli, M., Tarquini, S., Fornaciai, A., and Wörner, G. (2010). The regular shape of stratovolcanoes: a DEM-based morphometrical approach. Journal of Volcanology and Geothermal Research, 193(3-4), 171-181. doi: 10.1016/j. jvolgeores.2010.03.012.

Kereszturi, G., and Németh, K. (2012). Monogenetic basaltic volcanoes: genetic classification, growth, geomorphology and degradation. In: K. Németh (ed.). Updates in Volcanology, New Advances in Undertanding Volcanic Systems (pp. 3-89). Massey: INTECH Open Access Publisher.

Laeger, K., Halama, R., Hansteen, T., Savov, I.P., Murcia, H.F., Cortés, G.P., and Garbe-Schönberg, D. (2013). Crystallization conditions and petrogenesis of the lava dome from the $\sim 900$ years BP eruption of Cerro Machín Volcano, Colombia. Journal of South American Earth Sciences, 48, 193-208. doi: 10.1016/j.jsames.2013.09.009.

Martínez, T., Valencia, R., Ceballos, H., Narváez, M., Pulgarín, A., Correa, T., Navarro, A., Murcia, A., Zuluaga, M., Rueda, G., y Pardo, V. (2014). Geología y estratigrafía del Complejo Volcánico 
Nevado del Ruiz. Informe final, Bogotá Manizales - Popayán. Servicio Geológico Colombiano, 853p.

Méndez, R.A. (2001). Informe sobre la geología y estratigrafía de flujos piroclásticos asociados al Volcán Cerro Machín. INGEOMINAS, Informe Interno, inédito. Manizales.

Monsalve, H., y Mora, H. (2005). Esquema geodinámico regional para el noroccidente de Suramérica (modelo de subducción y desplazamientos relativos). Boletín de Geología, 27(44), 25-53.

Mosquera, D. (1978). Geología del cuadrángulo K-8. Manizales. INGEOMINAS, Informe 1763, Bogotá.

Murcia, H.F., Borrero, C.A., Pardo, N., Alvarado, G., Arnosio, M., y Scolamacchia, T. (2013). Depósitos volcaniclásticos: Términos y conceptos para una clasificación en español. Revista Geológica de América Central, 48, 15-39. doi: 10.15517/rgac. v0i48.12211.

Murcia, H.F., Hurtado, B.O., Cortés, G.P., Macías, J.L., y Cepeda, H. (2008). The 2500 yr B.P. Chicoral non-cohesive debris flow from Cerro Machín Volcano, Colombia. Journal of Volcanology and Geothermal Research, 171(3-4), 201-214. doi: 10.1016/j.jvolgeores.2007.11.016.

Murcia, H.F., Sheridan, M.F., Macias, J.L., and Cortés, G.P. (2010). TITAN2D simulations of pyroclastic flow at Cerro Machín Volcano, Colombia: Hazard implications. Journal of South American Earth Science, 29(2), 161-170. doi: 10.1016/j. jsames.2009.09.005.

Núñez, T.A., González, H., y Linares, E. (1979). Nuevas edades K/Ar de los esquistos verdes del Grupo Cajamarca. Publicaciones especiales de Geología. Universidad Nacional de Medellín, 23, $18 \mathrm{p}$.

Obando, M.G., y Ramos, R.A. (2003). Evaluación de la amenaza por flujos piroclásticos en la localidad de Cajamarca (Tolima) ante una probable erupción del Volcán Cerro Machín. Tesis, Universidad Nacional de Colombia, Bogotá, Colombia.

Pardo, N., Avellán, D.R., Macías, J.L., Scolamacchia, T., and Rodríguez, D. (2008). The $\sim 1245$ yr
BP Asososca maar: New advances on recent volcanic stratigraphy of Managua (Nicaragua) and hazard implications. Journal of Volcanology and Geothermal Research, 176(4), 493-512. doi: 10.1016/j.jvolgeores.2008.04.020.

Rueda, H. (2000). Depósitos de caída asociados a la actividad del volcán Cerro Machín: caracterización y evaluación de su amenaza potencial. Tesis, Universidad de Caldas, Manizales, Colombia.

Rueda, H. (2005). Erupciones plinianas del Holoceno en el volcán Cerro Machín, Colombia. Estratigrafía, petrografía y dinámica eruptiva. MSc Tesis, Universidad Nacional Autónoma de México, México D.F.

Scolamacchia, T., Macías, J.L., Sheridan, M.F., and Hughes, S.R. (2005). Morphology of ash aggregates from wet pyroclastic surges of the 1982 eruption of El Chichón Volcano, Mexico. Bulletin of Volcanology, 68(2), 171-200. doi: 10.1007/s00445-005-0430-x.

Trenkamp, R., Kellogg, J.N., Freymueller, J.T., and Mora, H. (2002). Wide plate margin deformation, southern Central America and northwestern South America, CASA GPS observations. Journal of South American Earth Sciences, 15(2), 157-171. doi: 10.1016/S0895-9811(02)00018-4.

Valentine, G.A., Graettinger, A.H., and Sonder, I. (2014). Explosion depths for phreatomagmatic eruptions. Geophysical Research Letters, 41(9), 3045-3051. doi: 10.1002/2014GL060096.

Van der Hilst, R., and Mann, P. (1994). Tectonic implications of tomographic images of subducted lithosphere beneath northwestern South America. Geology, 22, 451-454.

Villagómez, D., Spikings, R., Magna, T., Kammer, A., Winkler, W., and Beltrán, A. (2011). Geochronology, geochemistry and tectonic evolution of the Western and Central Cordilleras of Colombia. Lithos, 125(3-4), 875-896.

Wadell, H. (1933). Sphericity and roundness of rock particles. The Journal of Geology, 41(3), 310-331.

Wentworth, C.K., and Williams, H.(1932). The classification and terminology of the pyroclastic rocks. Bulletin of the National Research Council, 89, 19-53. 
Wohletz, K.H. (1986). Explosive magma-water interactions: Thermodynamics, explosion mechanisms, and field studies. Bulletin of Volcanology, 48(5), 245-264. doi: 10.1007/ BF01081754.

Wood, C.A. (1980). Morphometric evolution of cinder cones. Journal of Volcanology and Geothermal Research, 7(3-4), 387-413.

Zimanowski, B., Büttner, R., Dellino, P., White, J., and Wohletz, K. (2015). Magma-water interaction and phreatomagmatic fragmentation. In: $\mathrm{H}$. Sigurdsson, B. Houghton, S.R. McNutt, H. Rymer, J. Stix (eds.). The Encyclopedia of Volcanoes (pp. 631-648). 2nd edition, Academic Press, Elsevier, USA.

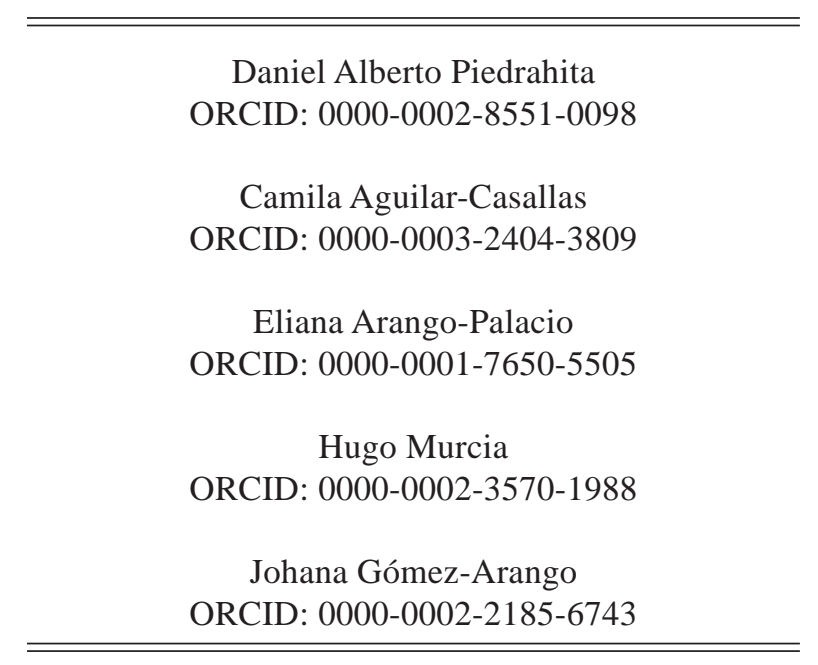

Trabajo recibido: diciembre 11 de 2017

Trabajo aceptado: julio 5 de 2018 